\title{
SPECTROSCOPIC BINARIES IN SOUTHERN OPEN CLUSTERS
}

\author{
H. Levato, ${ }^{1}$ J. F. González, ${ }^{1}$ S. Malaroda, ${ }^{1,2}$ and M. Grosso ${ }^{1}$ \\ RESUMEN
}

Aportamos un informe del trabajo sobre binarias que venimos desarrollando en cúmulos del hemisferio sur. El propósito es contribuir a comprender la formación y evolución de binarias espectroscópicas, proporcionando condiciones de contorno que permitan verificar algunas de las teorías actuales sobre la formación de binarias en cúmulos abiertos.

\section{ABSTRACT}

This is a report on an ongoing program about binaries in southern open clusters. The long-term purpose of this project is to contribute to understanding the formation and evolution of spectroscopic binaries, providing observational constraints that will permit tests of some of the current theories on binary formation in open clusters:

Key Words: BINARIES: SPECTROSCOPIC - GALAXY: OPEN CLUSTERS AND ASSOCIATIONS

\section{INTRODUCTION}

We would like to provide answers to the following general questions: 1) Is it true that the dense and compact open clusters can not have, among their members, hard spectroscopic binaries because if they do, the cluster would expand? 2) Why is the proportion of observed spectroscopic binaries different among open clusters? 3) Is it true that the spectroscopic binaries in open clusters are formed by dynamical encounters among their members?

Specifically this research is intended to answer the following particular questions: Is there a systematic variation of the frequencies of binaries in open clusters and associations or are the different frequencies observed simply a consequence of random fluctuations, inherent to the formation process of binaries? Is there a statistical relation between the periods of the binaries found in open clusters and the ages derived for them, in agreement with the current theory of formation of binaries through dynamical encounters among stars in open clusters? Is there a statistical relation between the mass ratios of the binaries found in open clusters and the age of these clusters, in agreement with the prediction of the same theory?

\section{RESEARCH DESIGN AND METHOD}

Essentially we measure radial velocities. If they vary with time we will conclude that the object observed is a binary. In some cases we will have two

\footnotetext{
${ }^{1}$ Complejo Astronómico El Leoncito, CONICET, San Juan, Argentina. hlevato@casleo.gov.ar

${ }^{2}$ Comisión de Investigaciones Científicas, Provincia de Bs. As., Argentina.
}

sets of lines in the spectrum so we will not have any doubt at all about the binary nature of the program object. The observation of the radial velocity variations with time permits derivation of the orbital parameters of the binary. In order to achieve this we are taking between 10 and 20 spectra per star. Cross-correlation and standard individual line measuring is being used for measuring the radial velocities. The resolution of the observations is around $0.14 \mathrm{~A} / \mathrm{px}$ in the blue portion of the spectra. We are trying to define the sample of stars to be observed in each cluster with similar complexities for binary detection (similar range of spectral type, similar range of $v \sin i$, etc). After reduction of the data using IRAF, radial velocities are derived, and the variations of this parameter are used to determine periods, and then preliminary orbits. Axial rotation is also determined. The ages of the open clusters are determined using the isochrones provided by Schaller (1992). In particular, to determine whether it is true that the compact open clusters do not have hard binaries among their members (see Penny et al. 1993) we are searching for radial velocity variations among members of young compact clusters. We will derive preliminary orbits when possible (generally the periods in these cases are on the order of a few days). Clusters of the Carina region, Orion and some Pismis are adequate for this purpose. In order to answer the second question, whether some orbital parameters of the binaries in open clusters will vary with the age of the clusters, we have defined three samples of clusters: one less than 20 millions years old, another with clusters between 100 and 400 million 
TABLE 1

CLUSTERS IN THE SAMPLE

\begin{tabular}{lcc}
\hline Cluster & Age & number of SB \\
\hline NGC 3532 & 8.40 & 5 \\
NGC 6025 & 7.75 & 7 \\
NGC 2527 & 8.83 & 4 \\
NGC 2362 & 6.77 & 5 \\
NGC 3766 & 7.42 & 1 \\
NGC 6281 & 8.45 & 1 \\
IC 4651 & 9.52 & 1 \\
NGC 6530 & 6.72 & 3 \\
NGC 2516 & 7.79 & 3 \\
NGC 2287 & 8.40 & 7 \\
NGC 2546 & 7.89 & 8 \\
TR 16 & 6.43 & 3 \\
Blanco 1 & 7.85 & 3 \\
\hline
\end{tabular}

years old and another sample with clusters around 1 billion years old. We will test the variation of the mass ratios, periods and synchronism with the age of the samples.

The third point to check is if the content of binaries in open clusters has certain systematic behavior with age or if the fluctuations are simply at random. In order to answer this point we will observe for binarity the same spectral range in a dozen clusters of different ages. The spectral range may be around late $\mathrm{A}$ or early $\mathrm{F}$ as a good compromise between availability of clusters in the sample, brightness of the stars to be observed and sensitivity of the electronic detectors. In all cases it will be desirable to derive preliminary orbits to confirm the binary nature of the radial velocity variations.

\section{OBSERVATIONAL DATA}

The observations are being obtained at Jorge Sahade telescope at CASLEO (Complejo Astronómico

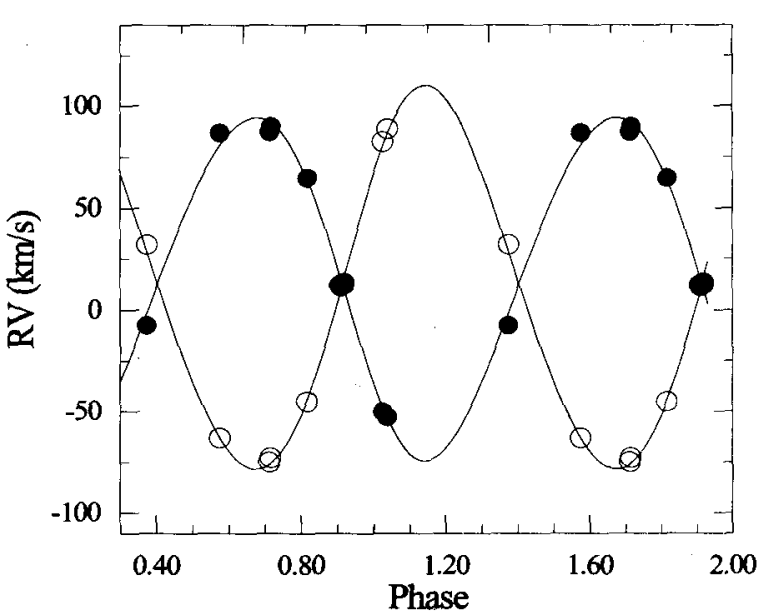

Fig. 1. Radial velocity curve for star 10 of NGC 6025 .

El Leoncito) using a REOSC echelle spectrograph at the Cassegrain focus. The detector used is a CCD TEK1024. The pixel size is $24 \mu$ and the 1-px resolution used is $0.14 \AA$ or $8 \mathrm{~km} / \mathrm{sec}$. The range of the spectra goes from $3800 \AA$ to $5400 \AA$ in 20 orders. Spectra were flatfielded and the wavelength calibration was done using a Th-Ar lamp. The reduction of the observational material is being done using IRAF package. Table 1 is a summary of the clusters observed up to now and the numbers of binaries in each one. As an example, Figure 1 shows the preliminary radial velocity curves of one spectroscopic binary detected in the cluster NGC 6025 .

We would like to thank CONICET (Consejo Nacional de Investigaciones Científicas y Técnicas) and the IAU for support.

\section{REFERENCES}

Penny, L., Gies, D., Hartkopf, W., Mason, B., \& Turner, N. 1993, PASP, 105, 588

Schaller, G., Schaerer, D., Meynet, G., \& Maeder, A. 1992, A\&AS, 96, 269 